\title{
Risk assessment of indeterminate lung nodule characterization by a novel plasma-protein multiplexed assay in current smokers: Results of a clinical experience program
}

\author{
Rami Arfoosh ${ }^{1}$, Kimtuyen Nguyen ${ }^{1}$, Amanda L Fish ${ }^{2 *}$, Veronica Wells ${ }^{2}$, Denise Rebeor ${ }^{2}$, Adam Seger ${ }^{2}$, Mike Beggs ${ }^{2}$, Gregory G Allen ${ }^{3}$, \\ Rabih El-Bizri ${ }^{3}$, Richard Torricelli ${ }^{3}$, DeEtta DeBault ${ }^{3}$, Angela Vallejo ${ }^{3}$, Jose Medina ${ }^{4}$, Rana Hasan6 , Kenneth Melby, David Miller ${ }^{6}$, Bryce $^{2}$ \\ Cowgill $^{6}$ and Thomas DeMarini ${ }^{6}$ \\ ${ }^{1}$ The Medical College of Georgia at Augusta University - Pulmonary and Sleep Specialists of Northeast Georgia, Hamilton Mill, Georgia, USA \\ ${ }^{2}$ MagArray Inc, Milpitas CA, USA \\ ${ }^{3}$ Roger Williams Medical Center, Providence, RI 02908, USA \\ ${ }^{4}$ Millennium Physician Group, Cape Coral, Florida, USA \\ ${ }^{5}$ Maranatha Medical Plaza, Valdosta, GA, USA \\ ${ }^{6}$ Southeastern Lung Care, Decatur, Georgia, USA
}

\begin{abstract}
Background: To reduce overdiagnosis and overtreatment of non-cancerous pulmonary nodules found on chest imaging, an accurate non-invasive and easily administered test is needed to assist in the detection and diagnosis of cancers in a cost-effective manner at an early stage, when curative interventions are still possible.

Objective: To assess the results of a novel, plasma-based multiplexed protein assay in a clinical experience program.

Methods: Fifty-four consecutive plasma samples were evaluated in a CLIA-certified laboratory using the novel blood test. All samples were from patients who are current smokers, aged 25 years and older, and have an indeterminate pulmonary nodule 0.4 to $3 \mathrm{~cm}$ in diameter.

Results: The mean patient age was 65.5 years and the mean nodule size was $1.0 \mathrm{~cm} .26$ patients were male (52\% female). Of the 54 tests, the assay results for 23 individuals were determined to be in the lower risk of malignancy range (score $\leq 49)$. 42 patients had a pre-test probability in the intermediate risk range as calculated by the VA Clinical Model. Of those patients, the assay characterized 22 as having a lower risk of malignancy (52\%).

Conclusion: The risk stratification of individuals with an indeterminate pulmonary nodule appears to be enhanced by identifying benign nodules compared to current methods in clinical practice. We hypothesize patients with benign disease may benefit the most from this assay by avoiding unnecessary subsequent overtreatment such as lung biopsy or bronchoscopy, while improving patient quality of care and reducing associated risks and costs from these procedures. Providers and their patients in whom they suspect lung cancer may consider using this novel assay prior to proceeding with more aggressive interventions.
\end{abstract}

\section{Introduction}

Lung nodule management decisions are based on the diagnostic yield of available tests to estimate the probability that a nodule is malignant. Clinical acumen and/or validated probability calculators are currently used to guide the individualized management of the patients' risk their nodule is malignant. These estimated risks can be grouped into low, intermediate, or high probability categories that relate to pathways for recommended management (Figure 1) [1].

Very small solid lung nodules have a low probability of malignancy and there are currently no tests available for these nodules with a useful diagnostic yield. Therefore, surveillance with CT imaging is recommended. Similarly, persistent sub-solid nodules often represent pre-invasive adenocarcinoma with very slow development to an invasive form. Because adjuvant imaging and non-surgical biopsies have a low yield in the diagnosis of sub-solid nodules, such nodules are often examined with CT imaging at intervals and for a duration that reflect their indolent nature.
Surgical resection provides a definitive diagnosis but is only used for lung nodules with a high probability of malignancy because surgery carries the largest potential for harm that is unnecessary for those with benign nodules.

Intermediate-risk nodules, defined by a probability of malignancy of 5-65\% using published risk calculators, constitute almost one-half of the pulmonary nodules identified by chest CT scan. These nodules require further diagnostic evaluation because of the concern for lung cancer. Adjuvant testing with positron emission tomography (PET)

${ }^{\star}$ Correspondence to: Amanda L Fish, MagArray Inc, Milpitas CA, USA, E-mail: Amanda.Fish@magarray.com

Key words: plasma-based multiplexed protein assay, lung nodule management, VA clinical factors model

Received: May 02, 2019; Accepted: May 15, 2019; Published: May 20, 2019 


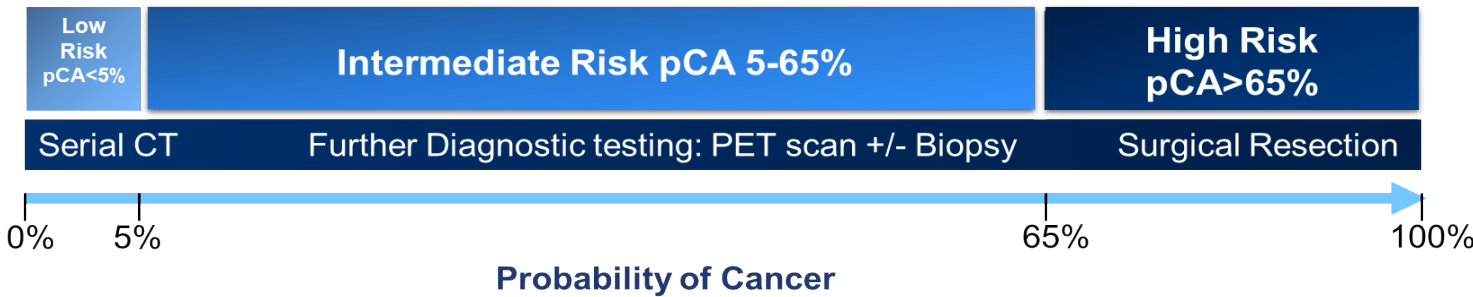

Figure 1. Estimating pre-test probability of cancer (pCA) facilitates clinical next steps

imaging or needle biopsy can help to characterize nodules with an intermediate probability of malignancy. However, the expense, modest specificity, and low yield of PET imaging, and the potential complications of needle biopsies affect the usefulness of these approaches. Even these minimally invasive procedures carry significant risks and cause anxiety to the patient, while the cost of a diagnostic evaluation increases 28 -fold when a biopsy is performed [2,3].

A recent publication highlights the clinical validation and performance of a novel, multiplexed, plasma protein signature as a risk assessment tool [4]. The authors established the assay's ability to aid in correctly identifying the risk of malignancy for a pulmonary nodule that falls into the inconclusive intermediate risk range for lung cancer as calculated by the VA Clinical Factors Model (VA model) [5]. In the independent validation cohort, 68 specimens had an intermediate risk pre-test probability of malignancy as calculated by the VA model. The biomarker algorithm-based test correctly identified $65 \%$ (44 specimens) of the intermediate-risk samples as having lower risk $(n=16)$ or higher risk $(n=28)$. The test showed a sensitivity of $94 \%$ and a negative predictive value (NPV) of $94 \%$ [4] in the intended use population having a cancer prevalence rate of $25 \%$ [6]. Almost all subjects (98\%) had early stage disease as defined by a lung cancer Stage I or II diagnosis [4].

The novel blood test demonstrated efficacy in accurately identifying patients at low risk of lung cancer to rule-out the need for risky and costly aggressive actions. That test has the potential to aid clinicians in more accurately characterizing radiologically indeterminate pulmonary nodules in current smokers and can help support a more informed clinical decision about performing an invasive evaluation of the patient's lung nodule [4].

\section{Materials and methods}

Plasma samples ( $\mathrm{K}_{2}$ EDTA) were obtained by a phlebotomist via venepuncture following the tube manufacturer's instructions and decanted into transport tubes for overnight shipment on icepacks to the CLIA-certified laboratory. All subsequent work was performed by staff in the CLIA-certified laboratory. Samples received at the lab were inspected for integrity and completeness of information. All 54 samples were accessioned into the laboratory database and then divided into nine aliquots ( 30 to $50 \mu \mathrm{L}$ ) with any additional sample remaining in the transport tube. The aliquots and transport tube were stored at $-80^{\circ} \mathrm{C}$. A sample aliquot was thawed for testing using the established procedures for the REVEAL test in the CLIA-certified laboratory, followed by data analysis to obtain the risk score as previously described [4]. Results of the assay were returned to the ordering clinician approximately one day after receiving the specimen in house.

\section{Results}

The demographic characteristics for the 54 plasma specimens from individual patients are summarized in table 1. REVEAL risk scores ranged from 8 to 94, with 23 samples identified as lower risk, and 31 identified as higher risk compared to the validated cut-off of 50 [8]. Using the VA Clinical Factors model, one sample had a low pre-test probability of cancer (pCA), 42 samples were calculated as having an intermediate risk pre-test probability of malignancy, and 11 samples were calculated as having a high risk pre-test probability of malignancy. Of the 42 samples having an intermediate risk pre-test probability of malignancy, 22 samples were lower risk by the novel blood test, and 20 were higher risk.

Interestingly, while the numbers of male and female samples are roughly equivalent in the intermediate risk pre-test probability of malignancy group, the novel blood test shows a higher proportion of female samples $(82 \%, 23 / 28$ samples) in the higher risk category as compared to male samples (31\%, $8 / 26$ samples). Relatively fewer female samples $(18 \%, 5 / 28)$ were identified in the lower risk category as compared to male samples ( $69 \%, 18 / 26$ samples). This is consistent with the overall nodule sizes presented for female subjects (mean 1.12, SD 0.74, range $0.4-3.0 \mathrm{~cm}$ ) generally being larger than those in the male subjects (mean 0.96, SD 0.51, range 0.4-2.5 cm). Of note, is that in both the lower risk category and the higher risk category, male subjects generally had larger nodules than female subjects, however the larger number of female subjects in the higher risk category made the average female subject nodule size greater than the average male subject nodule size.

Anecdotally, when combined with traditional risk factors, the Clinical Experience Program participating physicians reported that a lower-risk score provided the confidence they and the patient needed to adopt a serial surveillance approach. Similarly, higher-risk scores, in combination with the patient's comprehensive history and physical, encouraged the physician to recommend further evaluation.

\section{Discussion}

A large majority (96.4\%) of indeterminate pulmonary nodules (IPN) identified by screening chest CT are benign, yet current predictive tools to discriminate benign from malignant nodules are suboptimal, leading to a large number of more frequent follow-up CT scans and unnecessary invasive procedures with attendant morbidity and rare mortality, increased anxiety, and wasted healthcare spending -- up to $\$ 28$ billion/year in the United States. Incorrect evaluations of IPN result in harms that range from anxiety to a high rate of unnecessary thoracotomies for benign nodules, and missed chances for treatment during follow-up, resulting in death [7]. A new paradigm of thinking may thus be necessary. (Figure 2).

Recent results from a 344,510-patient study by Huo and colleagues [8] show that diagnostic procedures performed for pulmonary abnormalities are associated with a higher risk of complications than previously thought. In an era of advanced technologies and an aging population with an increasing number of chronic critically ill 
Arfoosh R (2019) Risk assessment of indeterminate lung nodule characterization by a novel plasma-protein multiplexed assay in current smokers: Results of a clinical experience program

Table 1. Sample set demographics

\begin{tabular}{|c|c|c|c|c|c|}
\hline & All Samples & $\begin{array}{c}\text { REVEAL Lower Risk }(< \\
\text { 50) }\end{array}$ & $\begin{array}{l}\text { REVEAL Higher Risk } \\
\qquad(\geq 50)\end{array}$ & $\begin{array}{c}\text { VA Pre-test } \\
\text { Probability } \\
\text { Intermediate }(565 \%)\end{array}$ & $\begin{array}{c}\text { VA Pre-test } \\
\text { Probability High } \\
(>65 \%)\end{array}$ \\
\hline \multicolumn{6}{|c|}{ Age, mean (SD) [range] years } \\
\hline Total & $65.3(10.3)[30-88]$ & $62.9(11.1)[30-79]$ & $67.1(9.6)[50-88]$ & $65.1(8.1)[44-79]$ & $69.2(12.5)[50-88]$ \\
\hline Male & $63.9(11.1)[30-81]$ & $62.8(11.5)[90-79]$ & $66.4(10.6)[53-81]$ & $64.5(8.5)[44-79]$ & $71.0(11.8)[58-81]$ \\
\hline Female & $66.6(9.6)[50-88]$ & $63.0(10.6)[51-75]$ & $67.4(9.4)[50-88]$ & $65.9(7.9)[51-78]$ & $68.5(13.4)[50-88]$ \\
\hline \multicolumn{6}{|c|}{ Nodule Size, mean (SD) [range] cm } \\
\hline Total & $1.05(.64)[0.4-3.0]$ & $0.67(0.28)[0.4-1.3]$ & $1.33(0.69)[0.48-3.0]$ & $0.82(0.39)[0.4-2.0]$ & $1.93(0.68)[0.9-3.0]$ \\
\hline Male & $0.96(0.51)[0.4-2.5]$ & $0.73(0.28)[0.4-1.3]$ & $1.48(0.55)[0.9-2.5]$ & $0.87(0.42)[0.4-2.0]$ & $1.70(0.70)[1.2-2.5]$ \\
\hline Female & $1.12(0.74)[0.4-3.0]$ & $0.44(0.05)[0.4-0.5]$ & $1.27(0.74)[0.48-3.0]$ & $0.76(0.35)[0.4-1.6]$ & $2.03(0.70)[0.9-3.0]$ \\
\hline \multicolumn{6}{|c|}{ VA Pretest Probability, mean (SD) [range] } \\
\hline Total & $48(19.9)[3-94]$ & $38(16.6)[3-59]$ & $57(17.2)[25-94]$ & $42(14.5)[8-65]$ & $75(8.4)[68-94]$ \\
\hline Male & $45(19.1)[3-82]$ & $37(16.9)[3-59]$ & $61(13.6)[42-82]$ & $42(14.8)[8-63]$ & $74(7.2)[68-82]$ \\
\hline Female & $51(20.5)[14-94]$ & $30(15.3)[14-48]$ & $56(18.5)[25-94]$ & $42(14.7)[14-65]$ & $76(9.3)[68-94]$ \\
\hline \multicolumn{6}{|c|}{ REVEAL Score, mean (SD) [range] } \\
\hline Total & $54(20.2)[8-94]$ & $35(10.3)[8-48]$ & $68(12.6)[50-94]$ & $48(15.4)[15-81]$ & $79(10.9)[57-94]$ \\
\hline Male & $44(17.6)[8-91]$ & $35(10.4)[8-48]$ & $64(13.0)[51-91]$ & $41(12.3)[15-68]$ & $73(17.1)[57-91]$ \\
\hline Female & $63(17.8)[22-94]$ & $36(11.4)[22-46]$ & $69(12.5)[50-94]$ & $56(15.1)[22-81]$ & $82(8.0)[68-94]$ \\
\hline \multicolumn{6}{|c|}{ Sample Numbers (n) } \\
\hline Total & 54 & 23 & 31 & 42 & 11 \\
\hline Male & 26 & 18 & 8 & 22 & 3 \\
\hline Female & 28 & 5 & 23 & 20 & 8 \\
\hline
\end{tabular}
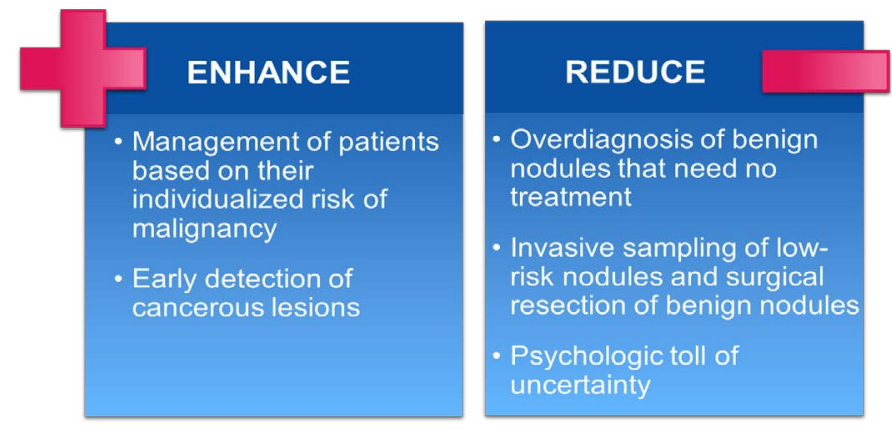

Figure 2. New paradigm

individuals, clinicians need to continue to carefully appraise the risk that may be incurred following a diagnostic procedure for a pulmonary lesion. Just as important to consider, are the benefits and diagnostic yield of a procedure, especially because the number of complications and associated costs have been shown to be higher in centers with a low volume of diagnostic procedures, or when there is an inability to provide a less-invasive procedure that can still deliver a diagnosis [8].

Deshwal and colleagues reported that bronchoscopy is of limited value, yet it is being performed frequently for lung nodule risk assessment [9]. The advent of newer technologies, including radial probe EBUS, virtual bronchoscopy, and electromagnetic navigation bronchoscopy, is likely driving the increased use of bronchoscopy in the evaluation of indeterminate pulmonary nodules. Although some studies have shown that a bronchoscopy guided by these tools has a higher diagnostic yield for IPN, a meta-analysis found the pooled diagnostic yield to be only $70 \%$. Those authors suggest that conventional bronchoscopy should be used carefully in the evaluation of peripheral nodules in an era expecting high-value and cost-effective care [9].

Another recent publication articulates the impact of an Interventional Pulmonology (IP) program and dedicated Pulmonary Nodule Clinic on surgical benign resection rates at a tertiary referral hospital. This state-of-the-art program was able to achieve a sustained reduction in benign pulmonary resections from $24 \%$ to $12 \%$, which the authors hypothesized was likely related to a decrease in diagnostic surgeries [10]. Such results underscore that predicting the risk of developing lung cancer is a difficult task and overdiagnosis is a serious problem. A more efficient and cost-effective strategy that would avoid delays in diagnosis, decrease radiation exposure, and reduce the need for invasive procedures would have a significantly positive impact on patient management [10].

Another important consideration when managing IPN is patient distress. Wiener and colleagues report that almost everyone with a nodule has a risk of suffering psychological harm. Most patients with IPN undergo radiographic surveillance rather than biopsy, leaving them in a state of uncertainly for months or years about whether they have cancer. Measured with the Impact of Event Scale, the authors identified $50 \%$ of the patients reported at least mild distress with $24 \%$ experiencing moderate or severe levels. They found clinicians underestimate patient distress or do not guide management decisions based on it. Moreover, most patients did not understand that surveillance is typically limited to 2 years; a poor understanding of the evaluation plan may also explain the finding that patients often were nonadherent to the surveillance plan [11].

Unlike aggressive diagnostic procedures, a non-invasive blood draw is safe, simple, and is not unduly influenced by the practitioner's skills. Once suspicious lesions are found by chest imaging, the identification of their properties could be further confirmed by a simple blood draw, with results returned a day after the sample is received by the CLIAcertified laboratory.

To address the large numbers of false positive findings, an effective rule-out test would be a valuable aid in the management of IPN. The performance reported for the novel plasma protein assay by Trivedi and colleagues [4] indicates that it could guide the clinician and their patient toward reassurance, watchful waiting, or sooner biopsy or resection, and thus decrease the anxiety, cost, overdiagnosis, and uncertainty typically associated with lung cancer screening. 
Trivedi and colleagues [4] articulated the false negative rate for the plasma-protein biomarker assay as $3 \%$. Even if patients receive a result indicating lower risk, it is unlikely they would be discharged completely from the practice. Rather, they will likely be asked to return for a follow up CT scan at a longer interval than might have been done in the absence of the test results. Similarly, a $33 \%$ false positive rate was reported by Trivedi. As previously mentioned, the novel assay was designed as a rule out test, and thus when the test indicates lower risk it can provide a very high level of confidence (NPV $=94 \%)$ to avoid unnecessary procedures. It is important to note that if the test indicates higher risk, other patient risk factors must be considered before deciding on an aggressive course of action. Establishing benignity can be a lengthy process (one to two years of radiographic stability). As well, invasive diagnostics also include the possibility of a false negative result.

A blood-based assay, such as the one described, is attractive due to the ease of acquisition. It is an accurate, timesaving, and cost effective way to help evaluate and characterize indeterminate pulmonary nodules, so clinicians and their patients can make more informed decisions about possible next steps.

In the clinical experience program presented, the blood-based test appears to provide important, additional information that can be used to modify a patient's management. This is especially critical for the patients with intermediate-risk nodules because they benefit from additional risk stratification tools to determine those who are in need of a more aggressive evaluation, and those for whom a less risky approach is warranted. Of the 42 samples having an intermediate risk pCA in the clinical experience program, the novel assay classified 22 samples as lower risk, and 20 samples as having higher risk. Thus, the risk assessment tool has the potential to aid clinicians in more accurately characterizing radiologically indeterminate pulmonary nodules in current smokers.

Based on the published performance as a 'rule out test', patients who receive a result indicating lower risk (test score of 49 or less out of 100) would have a high probability that their nodule is malignant [4]. That information may help patients and providers make a betterinformed decision to adopt a serial surveillance approach, avoiding the cost and risk of an invasive test, and reducing the psychologic toll of uncertainty.

\section{Limitations}

Although the blood-based biomarker assay has shown promising results in differentiating malignant from benign lesions, further research is needed to more broadly assess the impact of the test on clinical decision making [12]. Ideally, long-term follow up including the rate of lung cancer deaths prevented using this test is desired to further verify this as an effective risk assessment of lung cancer.

The plasma-protein signature should also be more directly assessed in all races, as well as specific conditions such as obesity and its pro-inflammatory state, steroid use, etc., that may affect the test performance. Finally, future clinical studies are warranted to further define the value of the test in accurately identify patients who are most likely to benefit from serial surveillance or early treatment, while reducing the rate of false-positive results, unnecessary interventions, and their associated morbidity and healthcare costs.

\section{Conclusions}

Here we report on the results of a clinical experience program. The novel, multiplexed, plasma-protein assay can be used as a non-invasive risk assessment tool by clinicians in characterizing indeterminate pulmonary nodules. When the results of this assay are combined with the traditional clinical risk factors (i.e. patient history), risk stratification for indeterminate pulmonary nodules may be improved compared to current methods in clinical practice. We hypothesize the assay will significantly reduce costs to the healthcare system while further improving a patient's quality of care. Providers and their patients may consider using this novel assay prior to proceeding with an invasive evaluation of their patient's indeterminate pulmonary nodule.

\section{Acknowledgments}

We are indebted to all clinicians and patients who participated in our Clinical Experience Program, as well as the entire MagArray, Inc., staff for their assistance in the laboratory.

\section{Disclosures}

MagArray, Inc., provided all financial support for the work reported in this article. No co-author has any financial disclosure or conflict of interest to report.

\section{References}

1. Mazzone PJ (2019) Molecular biomarkers for the evaluation of lung nodules. Lancet Respir Med 7: 297-298. [Crossref]

2. Lokhandwala T, Bittoni MA, Dann RA, D'Souza AO, Johnson M, et al. (2017) Costs of diagnostic assessment for lung cancer: A Medicare claims analysis. Clin Lung Cancer 18: e27-e34. [Crossref]

3. Gareen IF, Duan F, Greco EM, Snyder BS, Boiselle PM, et al. (2014) Impact of lung cancer screening results on participant health-related quality of life and state anxiety in the National Lung Screening Trial. Cancer 120: 3401-3409. [Crossref]

4. Trivedi NN, Arjomandi M, Brown JK, Rubenstein T, Rostykus AD, et al. (2018) Risk assessment for indeterminate pulmonary nodules using a novel, plasma-protein based biomarker assay. Biomed Res Clin Prac 3: 1-8

5. Gould MK, Ananth L, Barnett PG, Veterans Affairs SNAP Cooperative Study Group (2007) A Clinical Model to Estimate the Pretest Probability of Lung Cancer in Patients with Solitary Pulmonary Nodules. Chest 131: 383-388. [Crossref]

6. Tanner NT, Aggarwal J, Gould MK, Kearney P, Diette G, et al. (2015) Management of pulmonary nodules by community pulmonologists: A multicenter observational study. Chest 148: 1405-1414. [Crossref]

7. Massion PP, Walker RC (2014) Indeterminate Pulmonary Nodules: Risk for Having or for Developing Lung Cancer? Cancer Prev Res 7: 1173-1178. [Crossref]

8. Huo J, Xu Y, Sheu T, Volk RJ, Shih YT (2019) Complication Rates and Downstream Medical Costs Associated with Invasive Diagnostic Procedures for Lung Abnormalities in the Community Setting. JAMA Intern Med 179: 324-332. [Crossref]

9. Deshwal H, Avasarala SK, Ghosh S, Mehta AC (2019) Forbearance with Bronchoscopy A Review of Gratuitous Indications. Chest 155: 834-847. [Crossref]

10. Polcz M, Maiga A, Brown L, Deppen S, Montgomery C, et al. (2019) The Impact of an Interventional Pulmonary Program on Nontherapeutic Lung Resections. J Bronchology Interv Pulmonol [Epub ahead of print] [Crossref]

11. Wiener RS, Gould MK, Woloshin S, Schwartz LM, Clark JA (2012) The Thing is Not Knowing: Patients Perspectives on Surveillance of an Indeterminate Pulmonary Nodule. Health Expect 18: 355-365. [Crossref]

12. Mazzone PJ, Sears CR, Arenberg DA, Gaga M, Gould MK, et al. (2017) Evaluating molecular biomarkers for the early detection of lung cancer: When is a biomarker ready for clinical use? An Official American Thoracic Society Policy Statement. Am J Respir Crit Care Med 196: e15-e29. [Crossref]

Copyright: (C2019 Arfoosh R. This is an open-access article distributed under the terms of the Creative Commons Attribution License, which permits unrestricted use, distribution, and reproduction in any medium, provided the original author and source are credited. 